\title{
COMBINACIÓN DE METFORMINA FRENTE A INHIBIDORES DE LA DIPEPTIDILPEPTIDASA Y SULFONILUREAS EN EL TRATAMIENTO DE LA DIABETES TIPO 2: IMPACTO CLÍNICO Y ECONÓMICO
}

\author{
Antoni Sicras-Mainar ${ }^{1, a}$, Ruth Navarro-Artieda ${ }^{2, b}$
}

\begin{abstract}
RESUMEN
Objetivos. Determinar las repercusiones clínicas: adherencia, control metabólico, hipoglucemias y eventos cardiovasculares (ECV) y económicas (recursos y costes) de la combinación de metformina frente a IDPP4 (inhibidores dipeptidilpeptidasa-4) y sulfonilureas en pacientes con diabetes tipo 2. Materiales y métodos. Diseño observacional-multicéntrico de carácter retrospectivo. Se evaluaron pacientes $\geq 30$ años, en tratamiento con metformina y que iniciaron un segundo tratamiento antidiabético oral durante 2008-2009. Se establecieron 2 grupos de estudio: a) metformina+IDPP4 y b) metformina+sulfonilureas. Principales medidas: comorbilidad, control metabólico ( $\mathrm{HbA} 1 \mathrm{c}<7 \%)$, cumplimiento y complicaciones (hipoglucemias, ECV). El seguimiento se realizó durante 2 años. El modelo de costes diferenció los costes sanitarios directos (atención primaria/especializada) e indirectos (productividad laboral). Análisis estadístico: modelos de regresión logística y ANCOVA. Resultados. Se reclutaron 1405 pacientes (edad-media: 67,1 años; varones: 56,2\%). Un 37,0\% iniciaron un segundo tratamiento con IDPP4 y un $63,0 \%$ con sulfonilureas. A los dos años de seguimiento, los pacientes tratados con IDPP4 mostraron un mayor cumplimiento terapéutico $(70,3 \%$ vs. $60,6 \% ; p<0,001)$; mejor control-metabólico $(64,3 \%$ vs. $60,6 \% ; p<0,001)$ y menor proporción de hipoglucemias $(13,9 \%$ vs. $40,4 \% ; p<0,001$, respectivamente. El promedio/unitario de los costes totales corregidos fue de $2341 €$ vs. $2512 € ; p=0,038$. Las tasas de ECV e insuficiencia renal fueron del $3,7 \%$ vs. $6,4 \% ; p=0,027$. La vildagliptina fue el fármaco más utilizado entre los IDPP4. Conclusiones. Las sulfonilureas fueron los fármacos más utilizados para el tratamiento de la diabetes. Los pacientes en tratamiento con IDPP4 presentaron mayor cumplimiento y control de la diabetes, con menores tasas de hipoglucemias y ECV, ocasionando menores costes sanitarios.
\end{abstract}

Palabras clave: Diabetes mellitus; Hipoglucemia; Costos de la atención en salud; Enfermedades cardiovasculares (fuente: DeCS BIREME).

\section{COMBINATION THERAPY OF METFORMIN VS DIPEPTIDULPEPTIDASE INHIBITORS AND SULFONYLUREAS IN TYPE 2 DIABETES: CLINICAL AND ECONOMIC IMPACT}

\begin{abstract}
Objectives. Determine the clinical repurcussions of adherence, metabolic control, hypoglycemia and cardiovascular events (CVE) and economics (resources and costs) in the combination therapy of metformin vs DPP-4 (dipeptidyl peptidase-4) inhibitors and sulfonylureas in patients with type 2 diabetes. Materials and methods. Observational-multicenter and retrospective design. We evaluated patients $\geq 30$ years of age in treatment with metformin and who started a second oral antidiabetic treatment during 2008-2009. 2 study groups were established: a) metformin + DPP-4 inhibitors, and b) metformin + sulfonylurea. Main measures: comorbidity, metabolic control ( $\mathrm{HbA} 1 \mathrm{c}<7 \%$ ), compliance and complications (hypoglycemia, CVE). Follow up was conducted over two years. The cost model differentiated between direct healthcare costs (primary/ specialty care), and indirect costs (labor productivity). Statistical analysis: logistic regression and ANCOVA models. Results. 1,405 patients were recruited (average age 67.1 years old; $56.2 \%$ male). $37.0 \%$ started a second treatment with DPP-4 inhibitors, and $63.0 \%$ with sulfonylureas. After two years of follow up, patients treated with DPP-4 inhibitors showed greater treatment adherence ( $70.3 \%$ vs. $60.6 \% ; p<0.001)$; better metabolic control ( $64.3 \%$ vs. $60.6 \% ; p<0.001)$, and a lower proportion of hypoglycemia ( $13.9 \%$ vs. $40.4 \%$; $p<0.001$, respectively). The average/unit of adjusted total costs was $€ 2,341$ vs. $€ 2,512$; $p=0.038$. CVE and renal failure rates were $3.7 \%$ vs. $6.4 \% ; p=0.027$. Vildagliptin was the most used drug among DPP-4 inhibitors. Conclusions. Sulfonylureas were the most used drug for diabetes treatment. Patients treated with DPP-4 inhibitors had higher adherence and control of diabetes, with lower rates of hypoglycemia and CVE, resulting in lower healthcare costs.
\end{abstract}

Key words: Diabetes mellitus; Hypoglycemia; Health care costs; Cardiovascular diseases (source: MeSH NLM).

\section{INTRODUCCIÓN}

\footnotetext{
Dirección de planificación, Badalona Serveis Assistencials SA, Badalona, Barcelona

Documentación médica, Hospital Germans Trias i Pujol, Badalona, Barcelona.

Doctor en Medicina, Máster en Salud Pública; ${ }^{\mathrm{b}}$ especialista en documentación médica

Recibido: : 07-07-14 Aprobado: 19-11-14
}

Citar como: Sicras-Mainar A, Navarro-Artieda R. Combinación de metformina frente a inhibidores de la dipeptidilpeptidasa y sulfonilureas en el tratamiento de la diabetes tipo 2: impacto clínico y económico. Rev Peru Med Exp Salud Publica. 2014;31(4):626-34. 
La diabetes mellitus tipo 2 (DM2) es una enfermedad con un elevado impacto socio-sanitario, no solo como factor de riesgo cardiovascular, sino también por las complicaciones micro y macrovasculares que ocasiona ${ }^{(1,2)}$. La detección y control de los diferentes factores de riesgo, sigue siendo la estrategia fundamental para prevenir las enfermedades cardiovasculares ${ }^{(3)}$.

En la DM2, el tratamiento no farmacológico comprende tres aspectos básicos: plan de alimentación, ejercicio físico y hábitos saludables. El objetivo del tratamiento farmacológico es conseguir un control metabólico óptimo con la máxima eficacia y seguridad posible ${ }^{(4,5)}$. En este aspecto, las sociedades científicas recomiendan el uso de metformina como fármaco de primera elección en las primeras fases del tratamiento y cuando el control glucémico no es adecuado en monoterapia, añadir un segundo fármaco (doble terapia), que hemos de adaptar de forma individualizada a cada paciente, teniendo en cuenta su edad, el nivel de hiperglucemia, el momento evolutivo de su enfermedad, las comorbilidades asociadas y la situación social y personal ${ }^{(6,8)}$. Diversos estudios ponen de manifiesto que la complicación aguda más frecuente en la DM2 es la hipoglucemia, especialmente en los pacientes tratados con insulina $\mathrm{y} / \mathrm{o}$ sulfonilureas (SU) ${ }^{(9,10)}$. La aparición de los inhibidores de la dipeptidil peptidasa 4 (IDPP4) presentan la potencial ventaja, frente a los secretagogos clásicos, de reducir las hipoglucemias, dado que su mecanismo estimulador de la secreción de insulina es dependiente de la glucosa ${ }^{(11)}$.

La DM2 es considerada como un problema de salud pública por sus repercusiones en la calidad de vida, y el elevado consumo de los recursos sanitarios ${ }^{(12,13)}$. Los estudios disponibles en práctica clínica sobre las complicaciones, el control metabólico y el uso de los recursos sanitarios y sus costes en pacientes en doble terapia farmacológica con antidiabéticos orales son limitados. El objetivo del estudio fue contrastar las repercusiones clínicas (adherencia al tratamiento, control metabólico, hipoglucemias y complicaciones macrovasculares) y económicas (uso de recursos y sus costes) en la combinación de metformina frente a IDPP4 y SU en pacientes con DM2 en situación de práctica clínica habitual, y en un ámbito poblacional.

\section{MATERIALES Y MÉTODOS}

\section{DISEÑO Y POBLACIÓN DE ESTUDIO}

Se realizó un estudio observacional, elaborado a partir de la revisión de los registros médicos (bases de datos informatizadas) de pacientes seguidos en régimen ambulatorio y hospitalario. La población de estudio estuvo formada por pacientes pertenecientes a seis centros de atención primaria (AP) y dos centros hospitalarios: Hospital Municipal y Hospital Universitari Germans Trías i Pujol de de Badalona. La población asignada a los centros fue en su mayoría urbana, de nivel socioeconómico medio-bajo, con predominio industrial. Se seleccionaron pacientes que frecuentaron los dos niveles asistenciales, tanto atención primaria y/o hospitalaria, con el propósito de tener un seguimiento más completo (continuum asistencial).

\section{CRITERIOS DE INCLUSIÓN Y EXCLUSIÓN}

Se incluyeron en el estudio a todos los pacientes que iniciaron un segundo tratamiento antidiabético oral (SU, IDPP4) entre 2008-2009 y que reunían las siguientes características: a) edad $\geq 30$ años, b) pacientes diagnosticados de DM2 un mínimo de 12 meses antes del inicio del estudio, c) seguir de forma regular el protocolo/ guía de riesgo cardiovascular establecido en los centros, d) estar en el programa de prescripciones crónicas para la obtención de las recetas médicas (con registro constatado de la dosis diaria, el intervalo de tiempo y la duración de cada tratamiento administrado), e) pacientes en tratamiento actual con metformina como primera opción terapéutica (monoterapia), y f) que se pudiera garantizar el seguimiento de los pacientes durante un periodo de dos años. Fueron excluidos los sujetos trasladados a otros municipios y los desplazados o fuera de zona.

\section{DEFINICIÓN DE DIABETES TIPO 2, VARIABLES SOCIODEMOGRÁFICAS Y DE COMORBILIDAD (VARIABLES BASALES)}

El diagnóstico de DM2 se obtuvo a partir de la Clasificación Internacional de la AP (CIAP-2; T90) ${ }^{(14)}$, y de la codificación de los ingresos y urgencias hospitalarias, según la Clasificación Internacional de Enfermedades, 9. ${ }^{a}$ revisión, modificación clínica; CIE-9-MC [250.00-250.92]). Se obtuvo información basal de las complicaciones microvasculares: a) retinopatía diabética, b) nefropatía diabética, c) neuropatía diabética y d) vasculopatía diabética. Se obtuvo además, la edad (continua), sexo, el tiempo de evolución de la DM2 y un conjunto de antecedentes personales obtenidos a partir de la CIAP-2 ${ }^{(14)}$. Como variable resumen de la comorbilidad general, para cada paciente atendido se utilizó: a) el índice de comorbilidad de Charlson ${ }^{(15)}$ como una aproximación a la gravedad del paciente.

\section{CUMPLIMIENTO, PERSISTENCIA AL TRATAMIENTO Y CONTROL METABÓLICO (VARIABLES INTERMEDIAS)}

Se obtuvo la información de los siguientes antidiabéticos orales según la Anatomical Therapeutic Chemical Classification System (ATC) ${ }^{(16)}$ : a) metformina (A10BA*);

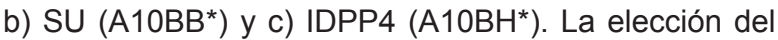


medicamento a un paciente en concreto fue a criterio del médico (práctica clínica). El cumplimiento del período se calculó mediante el cociente entre el número total de comprimidos dispensados y los recomendados o prescritos. En este aspecto, el cociente entre la medicación dispensada (oficinas de farmacia) entre la prescrita (médico) debe interpretarse como una variable "proxy" de la adherencia, dado que no es posible saber si el paciente tomó con certeza todos los comprimidos dispensados. La dispensación farmacológica se obtuvo de la información emitida por la aplicación (RCMPS) del CatSalut. La persistencia del tratamiento se definió como el tiempo, medido en meses, sin abandono del tratamiento inicial o sin cambio a otra medicación a partir de los 60 días después de la prescripción inicial (17). El control metabólico se estableció a partir de niveles de hemoglobina glicosilada $(\mathrm{HbA} 1 \mathrm{c})<7 \%{ }^{(2)}$.

\section{COMPLICACIONES ACONTECIDAS DURANTE EL SEGUIMIENTO DE LOS PACIENTES (VARIABLES DE RESULTADO)}

También se identificaron complicaciones ocurridas durante el seguimiento de los pacientes. Estos fueron: a) los casos de hipoglucemia sintomática, y b) macrovasculares o eventos cardiovasculares (ECV) e insuficiencia renal. Estas últimas incluyeron: a) cardiopatías, como la isquemia cardiaca, el infarto agudo de miocardio y la insuficiencia cardíaca, según la definición de los criterios diagnósticos de la Organización Mundial de la Salud; b) enfermedad cerebrovascular, como el ictus (isquémico o hemorrágico según la American Heart Association) (1) y el accidente isquémico transitorio; c) la enfermedad arterial periférica (todos los tipos), y d) la enfermedad renal (nefropatía diabética o deterioro de la función renal [creatinina sérica: varones > 133; mujeres > 124 mmol o filtrado glomerular $<60 \mathrm{~mL} / \mathrm{min}]$ ). La tasa de incidencia acumulada fue definida como la proporción de individuos sanos que desarrollaron la complicación (número de casos nuevos).

\section{USO DE RECURSOS Y MODELO DE COSTES (VARIABLES DE RESULTADO)}

Se consideraron como costes directos sanitarios los relacionados con la actividad asistencial efectuadas por los profesionales y como costes no sanitarios o indirectos, los relativos a las pérdidas de productividad laboral (número de bajas y días de incapacidad). Los diferentes conceptos de estudio y su valoración económica se detallan en la Tabla 1 (correspondientes al año 2011). Las diferentes tarifas se obtuvieron a partir de la contabilidad analítica de los centros, excepto la medicación y los días de baja laboral. Los días de incapacidad laboral o pérdidas de productividad, se consideraron como costes
Tabla 1. Detalle de los costes unitarios y de las pérdidas de productividad laboral

\begin{tabular}{lc}
\hline Recursos sanitarios y no sanitarios & $\begin{array}{c}\text { Costes unitarios } \\
(\boldsymbol{\epsilon})\end{array}$ \\
\hline Visitas médicas & \\
Visita médica a atención primaria & 23,19 \\
\hline Visita médica a urgencias & 117,53 \\
\hline Hospitalización (un día) & 320,9 \\
\hline Visita médica a atención especializada & 104,41 \\
Pruebas complementarias & \\
\hline Pruebas de laboratorio & 22,3 \\
\hline Radiología convencional & 18,5 \\
\hline Pruebas diagnósticas/terapéuticas & 37,12 \\
Prescripción farmacéutica & PVPiva \\
Productividad laboral - costes indirectos & \\
\hline Coste por día no trabajado & 101,21 \\
\hline
\end{tabular}

Fuente de los recursos sanitarios: contabilidad analítica propia. Valores expresados en euros. PVPiva: precio de venta al público con IVA. $\left(^{*}\right)$ fuente Instituto Nacional de Estadística. Costes pertenecientes al año 2011

no sanitarios (costes indirectos). El coste se cuantificó según el salario mínimo interprofesional (fuente: INE) ${ }^{(18)}$.

\section{ANÁLISIS ESTADÍSTICO}

Se efectuó un análisis estadístico descriptivo-univariante con valores de media, desviación típica/estándar (DE) e intervalos de confianza (IC) del $95 \%$ y se comprobó la normalidad de la distribución con la prueba de Kolmogorov-Smirnov para las variables cuantitativas. En el análisis bivariante se utilizaron las pruebas de la ANOVA, chi cuadrado, correlación lineal de Pearson y no paramétrica de Mann-Whitney-Wilcoxon. Se efectuó un análisis de regresión logística para determinar las comorbilidades asociadas al grupo de IDPP4, y otro para definir las variables asociadas al ECV (presencia/ ausencia), con procedimiento enter (estadístico: Wald). La comparación del coste ambulatorio y hospitalario se realizó según las recomendaciones de Thompson y Barber (19) mediante el análisis de la covarianza (ANCOVA), con el sexo, la edad y el índice de Charlson como covariables (procedimiento: estimación de medias marginales, ajuste de Bonferroni).

\section{CONSIDERACIONES ÉTICAS}

Se respetó la confidencialidad de los registros marcada por la Ley Orgánica de Protección de Datos (15/1999, de 13 de diciembre), mediante datos disociados. El estudio fue clasificado por la Agencia española del medicamento y Productos Sanitarios (No-EPA), y posteriormente fue aprobado por el Comité de Ética de Investigación Clínica del Hospital Clínico y Provincial de Barcelona.

\section{RESULTADOS}


De una selección de 62370 sujetos mayores de 30 años asignados y atendidos regularmente en los centros, 48 295 demandaron atención y 6620 pacientes estaban diagnosticados de DM2 (prevalencia: 10,6\%; IC 95\%: 10,4-10,8). Fueron excluidos del estudio 4553 pacientes; 978 no recibían tratamiento farmacológico, 464 recibieron otras terapias farmacológicas, 241 suspendieron el tratamiento, 1887 modificaron la terapia durante el seguimiento, 655 se consideraron pérdidas durante el seguimiento y 328 por causas no conocidas y/o otras causas. La distribución porcentual de los pacientes excluidos según los dos grupos de estudio fue similar.

Se seleccionaron para el estudio 1405 pacientes en tratamiento con doble terapia. Por grupos de estudio, un $63,0 \%(\mathrm{~N}=886)$ con $\mathrm{SU}$ y un $37,0 \%(\mathrm{~N}=519)$ con IDPP4. El consumo de SU fue el siguiente: glibenclamida $(66,5 \%)$, gliclazida $(15,8 \%)$, glimepirida $(10,2 \%)$, glipizida $(5,2 \%)$ y otras SU (2,3\%). En general, la media de edad fue de 67,1 (DE: 10,8) años y el $56,2 \%$ fueron varones. En la Tabla 2 se describen las características basales de la serie estudiada

Tabla 2. Características basales de la serie estudiada

\begin{tabular}{|c|c|c|c|}
\hline & $\begin{array}{l}\text { Metformina } \\
+ \text { SU }\end{array}$ & $\begin{array}{l}\text { Metformina } \\
+ \text { IDPP4 }\end{array}$ & \multirow{2}{*}{$p$} \\
\hline & $\mathrm{N}=886$ (\%) & $\mathrm{N}=519(\%)$ & \\
\hline \multicolumn{4}{|c|}{ Características sociodemográficas } \\
\hline Promedio de edad en años* & $67,8(11)$ & $65,8(10,4)$ & 0,001 \\
\hline Hombres & $(57,4)$ & $(53,9)$ & 0,202 \\
\hline \multicolumn{4}{|l|}{ Comorbilidad general } \\
\hline Promedio de diagnósticos ${ }^{*}$ & $5,4(2,4)$ & $5,3(2,3)$ & 0,479 \\
\hline Promedio índice de Charlson* & $1,0(0,4)$ & $1,0(0,5)$ & 0,910 \\
\hline \multicolumn{4}{|l|}{ Comorbilidades asociadas } \\
\hline Hipertensión arterial & $(69,4)$ & $(65,9)$ & 0,172 \\
\hline Dislipemia & $(64,6)$ & $(64,0)$ & 0,823 \\
\hline Obesidad & $(24,2)$ & $(26,2)$ & 0,391 \\
\hline Fumadores activos & $(20,1)$ & $(23,5)$ & 0,131 \\
\hline Alcoholismo & $(3,8)$ & $(2,7)$ & 0,256 \\
\hline Cardiopatía isquémica & $(14,1)$ & $(12,9)$ & 0,528 \\
\hline Accidente vásculocerebral & $(16,0)$ & $(13,1)$ & 0,138 \\
\hline Insuficiencias orgánicas & $(16,9)$ & $(16,4)$ & 0,789 \\
\hline Asma bronquial & $(4,1)$ & $(4,4)$ & 0,740 \\
\hline EPOC & $(5,5)$ & $(6,9)$ & 0,286 \\
\hline Neuropatías & $(0,7)$ & $(0,8)$ & 0,841 \\
\hline Demencias (todos los tipos) & $(3,4)$ & $(2,7)$ & 0,475 \\
\hline Síndrome depresivo & $(17,9)$ & $(18,7)$ & 0,559 \\
\hline Neoplasias malignas & $(9,7)$ & $(8,9)$ & 0,668 \\
\hline \multicolumn{4}{|l|}{ Relación con la diabetes } \\
\hline $\begin{array}{l}\text { Tiempo desde el diagnóstico, } \\
\text { años }^{*}\end{array}$ & $8,3(11,3)$ & $8,9(4,6)$ & 0,222 \\
\hline Retinopatía diabético & $(14,6)$ & $(17,0)$ & 0,230 \\
\hline Neuropatía diabética & $(2,50)$ & $(3,1)$ & 0,504 \\
\hline Nefropatía diabética & $(1,90)$ & $(1,7)$ & 0,804 \\
\hline Alteración del filtrado glomerular & $(14,6)$ & $(12,1)$ & 0,202 \\
\hline
\end{tabular}

Tabla 3. Relación entre el cumplimiento, persistencia y grado de control de la diabetes. Seguimiento a los dos años

\begin{tabular}{|c|c|c|c|}
\hline & $\begin{array}{l}\text { Metformina } \\
+ \text { SU }\end{array}$ & $\begin{array}{l}\text { Metformina } \\
+ \text { IDPP4 } \\
\end{array}$ & $p$ \\
\hline & $\mathrm{N}=886$ & $\mathrm{~N}=519$ & \\
\hline $\begin{array}{l}\text { Tiempo con metformina en } \\
\text { meses* }^{*}\end{array}$ & $8,3(11,3)$ & $8,9(4,6)$ & 0,222 \\
\hline Uso de metformina > 1 año & $(75,9)$ & $(74,6)$ & 0,434 \\
\hline Cumplimiento terapéutico & $(60,6)$ & $(70,3)$ & $<0,001$ \\
\hline$\geq 80 \%$, alto & $(40,1)$ & $(55,1)$ & \\
\hline $50-79 \%$, medio & $(34,7)$ & $(29,1)$ & \\
\hline$<50 \%$, bajo & $(25,2)$ & $(15,8)$ & $<0,001$ \\
\hline Persistencia del tratamiento & $(52,4)$ & $(63,4)$ & $<0,001$ \\
\hline Control óptimo de la DM2 & & & \\
\hline Periodo inicial $^{* *}$ & $(59,2)$ & $(61,2)$ & 0,463 \\
\hline Periodo final ${ }^{\star * *}$ & $(60,6)$ & $(64,3)$ & 0,031 \\
\hline Diferencia de los porcentajes & $(1,4)$ & $(3,1)$ & 0,041 \\
\hline
\end{tabular}

* media (desviación estándar). Cumplimiento terapéutico: relación entre el número de comprimidos dispensados entre los prescritos. Persistencia: tiempo sin abandono del tratamiento inicial o sin cambio a otra medicación a partir de los 60 después de la prescripción inicial. Control óptimo: niveles de $\mathrm{HbA} 1 \mathrm{c}<7 \%$. SU: sulfonilureas; IDPP4: inhibidores de la dipeptilpeptidasa 4.

** Periodo inicial: momento de inclusión del segundo medicamento (SU o IDPP4).

*** Periodo final: finalización del seguimiento de los pacientes (dos años).

según los grupos de pacientes. Los sujetos en tratamiento con IDPP4 en comparación con SU mostraron una media de edad inferior respecto al grupo de referencia $(65,8$ frente a 67,8 años, $p=0,001$ ) y una similar comorbilidad general (5,3 frente a 5,4 diagnósticos, respectivamente). Las comorbilidades específicas y las complicaciones microvasculares no mostraron diferencias destacables.

Los pacientes del grupo de IDPP4 mostraron un mejor cumplimiento terapéutico $(70,3 \%$ frente al $60,6 \%$; $p<0,001)$ y persistencia del tratamiento $(63,4 \%$ frente al $52,4 \% ; p<0,001)$ respectivamente, Tabla 3 . Se obtuvo una aceptable correlación entre el grado de cumplimiento y el tiempo en meses de persistencia al tratamiento $(r=0,433$; $p<0,001)$. Al inicio de la terapia dual no se obtuvieron diferencias significativas entre los niveles de $\mathrm{HbA} 1 \mathrm{c}$ en los dos grupos analizados (IDDP4 vs. SU: $8,2 \%$ frente al $8,6 \% ; p=0,793)$. El control metabólico $(\mathrm{HbA} 1 \mathrm{c}<7 \%)$ de la DM2 en el grupo de IDPP4 al finalizar el seguimiento de los pacientes fue superior $(64,3 \%$ frente al $60,6 \%$ respectivamente; $p=0,031)$. En el modelo logístico, los pacientes en tratamiento con IDPP4 se relacionaron (asociación) con el cumplimiento terapéutico OR=1,3 (IC $95 \%: 1,1-1,5 ; p=0,021)$, la persistencia del tratamiento OR=1,2 (IC 95\%: 1,1-1,4; $p=0,022)$ y el control metabólico $\mathrm{OR}=1,3$ (IC 95\%: 1,1-1,6; $p=0,033)$.

Los sujetos con uso de IDPP4 en comparación con SU consumieron menos visitas médicas en AP (21,6 frente a $28,9 ; p<0,001)$, menos visitas en atención especializada $(1,8$ frente a 2,$3 ; p=0,009)$ y menos días de incapacidad 
Tabla 4. Modelo de costes brutos y corregidos (seguimiento de 24 meses) según los grupos de estudio en una cohorte retrospectiva (en EUR)

\begin{tabular}{|c|c|c|c|c|}
\hline & Metformina + SU & Metformina + IDPP4 & Total & \multirow{2}{*}{$p$} \\
\hline & $\mathrm{N}=886$ & $\mathrm{~N}=519$ & $N=1405$ & \\
\hline \multicolumn{5}{|l|}{ Modelo de costes sin corregir } \\
\hline Costes sanitarios & $2.416,4(1.391,2)$ & $2.241,8(1.161,7)$ & $2.351,9(1.313,4)$ & 0,016 \\
\hline Costes en atención primaria & $2.132(1.198,5)$ & $2.016,9(1.071,7)$ & $2.089,4(1.154,2)$ & 0,071 \\
\hline Visitas médicas & $669,5(349,2)$ & $501,9(343,0)$ & $607,6(356,1)$ & $<0,001$ \\
\hline Pruebas de laboratorio & $67,5(40,6)$ & $49,5(33,2)$ & $60,9(39,0)$ & $<0,001$ \\
\hline Radiología convencional & $18,0(22,9)$ & $19,7(22,8)$ & $18,6(22,9)$ & 0,179 \\
\hline Pruebas complementarias & $17,1(27,8)$ & $17,0(25,3)$ & $17,0(26,9)$ & 0,946 \\
\hline Medicamentos & $1.360(998,6)$ & $1.428,8(893,3)$ & $1.385,4(961,3)$ & 0,195 \\
\hline - Metformina & $468,9(315,2)$ & $300,2(224,1)$ & $420,8(328,9)$ & 0,035 \\
\hline - SU vs. IDPP4 & $640,2(259,6)$ & $975,5(429,7)$ & $737,5(325,5)$ & 0,022 \\
\hline - Otros fármacos & $251,1(187,2)$ & $152,3(101,1)$ & $226,7(157,9)$ & 0,328 \\
\hline Costes en atención especializada & $284,4(457,8)$ & $225(275,4)$ & $262,5(401,1)$ & 0,007 \\
\hline Días de hospitalización & $16,7(233,3)$ & $6,2(52,4)$ & $12,8(188,0)$ & 0,313 \\
\hline Visitas médicas & $238,9(369,9)$ & $190,9(252,0)$ & $221,2(332,0)$ & 0,009 \\
\hline Urgencias & $28,9(60,0)$ & $27,9(63,2)$ & $28,5(61,2)$ & 0,753 \\
\hline Costes no sanitarios (productividad) & $57,6(511,8)$ & $1,1(0,1)$ & $36,3(407,3)$ & 0,011 \\
\hline Costes totales & $2.474,0(1.471,8)$ & $2.241,8(1.161,7)$ & $2.388,2(1.369,6)$ & 0,002 \\
\hline Modelo de costes corregidos* & & & Diferencia & \\
\hline Costes sanitarios (IC 95\%) & $2.454,9(2.324,3-2.485,4)$ & $2.332,9(2.227,1-2.438,7)$ & -122 & 0,031 \\
\hline Costes en atención primaria (IC 95\%) & $2.173,1(2.052,5-2.193,7)$ & $2.097,4(2.004,7-2.190,1)$ & $-75,7$ & 0,666 \\
\hline Costes en atención especializada (IC 95\%) & $281,8(254,5-308,9)$ & $235,5(199,7-271,2)$ & $-46,3$ & 0,044 \\
\hline Costes no sanitarios (productividad) (IC 95\%) & $57,6(29,6-85,5)$ & $8(0,1-35,9)$ & $-49,6$ & 0,013 \\
\hline Costes totales (IC 95\%) & $2.512,4(2.377,8-2.547,1)$ & $2.340,9(2.220,9-2.443,3)$ & $-171,5$ & 0,038 \\
\hline
\end{tabular}

Valores expresados en media (desviación estándar); p: significación estadística; IC: intervalo de confianza; $\left({ }^{*}\right)$ Modelo de ANCOVA: los contrastes se basan en las comparaciones por pares, linealmente independientes, entre las medias marginales estimadas. Co-variables: edad e índice de Charlson. Componentes fijos: sexo y grupos de fármacos. SU: sulfonilureas; IDPP4: inhibidores de la dipeptilpeptidasa 4.

laboral $(0,1$ frente a 0,$6 ; p=0,011)$. El modelo de costes brutos y corregidos (covariables), seguimiento de dos años, según los grupos de estudio se describe en la Tabla 4. El coste total de la atención de los pacientes diabéticos, ascendió a 3,3 millones de euros, de los cuales el $98,5 \%$ correspondieron a costes sanitarios directos y el $1,5 \%$ a costes no sanitarios indirectos. El promedio/unitario de los costes totales (sanitarios y no sanitarios) de los sujetos del grupo de IDPP4 en comparación con SU fueron inferiores (2242 € frente a $2474 € ; p=0,002$ ). En el modelo corregido (ANCOVA) fue de $2341 €$ (IC 95\%: 2221-2443) frente a $2512 €$; (IC 95\%: 2378-2547); $p=0,038$. El coste sanitario mostró una moderada correlación con la edad $(r=0,344)$ y la comorbilidad general $r=0,352), p<0,001$.

Las tasas de ECV e insuficiencia renal se detallan en la Figura 1. En general, el número de pacientes con ECV fue de 76 (tasa: 5,4\%; IC 95\%: 4,4-6,4). La tasa de ECV del grupo tratado con IDPP4, en comparación con el de SU fueron inferiores $(3,7 \%$ frente al $6,4 \%, p=0,027)$. En el modelo de regresión logística, la presencia de ECV se asoció al incumplimiento terapéutico OR=1,1 (IC 95\%: $1,0-1,5)$, al inadecuado control metabólico de la DM2 OR=1,2 (IC 95\%: 1,1-1,5), al sexo masculino OR=1,3
(IC 95\%: 1,1-2,1) y a la edad OR=1,1 (IC 95\%: 1,0-1,2), $p<0,05$. El porcentaje de pacientes con hipoglucemias fue del $30,6 \%$. Los pacientes del grupo de IDPP4 presentaron una menor proporción de hipoglucemias $(13,9 \%$ frente al $40,4 \%, p<0,001)$. En general, el $0,7 \%$

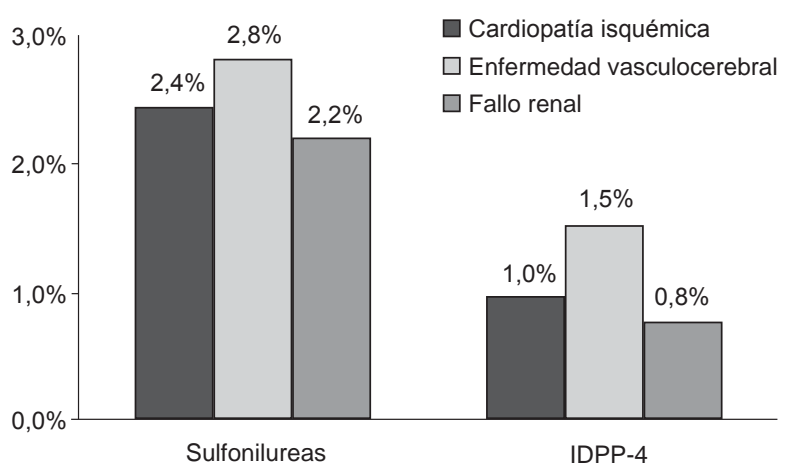

Un paciente pudo tener más de un evento. Insuficiencia renal: corresponde a un filtrado glomerular $<60 \mathrm{~mL} / \mathrm{min}$. Diferencias significativas en las comparaciones por pares $(p<0,05)$. Combinación de metformina con sulfonilureas y metformina con DPP-4 (inhibidores de la dipeptilpeptidasa).

Figura 1. Distribución de los eventos cardiovasculares según los grupos de estudio durante los dos años de seguimiento. 
de los pacientes requirieron un ingreso hospitalario, el $1,2 \%$ se atendieron en urgencias hospitalarias y el $29,7 \%$ en AP.

La Vildagliptina fue el fármaco más utilizado entre los IDPP4 $(80,1 \%)$. En el subanálisis, los resultados, en cuanto a cumplimiento $(71,1 \%)$, persistencia $(62,7 \%)$, control metabólico $(65,3 \%)$, hipoglucemias $(13,2 \%)$ y complicaciones macrovasculares $(3,5 \%)$, fueron similares a los obtenidos en el grupo de IDPP4, y también mostraron diferencias estadísticamente significativas con respecto al grupo de SU $(p<0,05)$.

\section{DISCUSIÓN}

Este estudio pone de manifiesto que los pacientes tratados con doble terapia con metformina y IDPP4 en comparación con metformina y SU, presentan menores tasas de complicaciones clínicas (hipoglucemias, ECV) y de costes sanitarios. En España es escasa la evidencia disponible que evalúe estas medidas en un solo estudio, por lo que esta circunstancia puede dar al estudio un cierto atractivo conceptual y de abordaje más completo. No obstante, cabe destacar que sin una adecuada estandarización de las metodologías en cuanto a las características de los pacientes, así como en el número y medida de las variables estudiadas, los resultados obtenidos deben de interpretarse con prudencia, obligando a ser cautelosos en la validez externa de los resultados.

No todos los pacientes con DM2 iniciaron un primer tratamiento farmacológico con metformina, pero de los que lo hicieron, las SU fueron los fármacos más utilizados como segunda opción terapéutica. En el estudio parece que las recomendaciones de las sociedades científicas se siguieron, aunque el inicio del tratamiento farmacológico fue más conservador (Tabla $3)^{(1,2,5)}$. Los resultados de este estudio revelan que a los dos años de seguimiento los pacientes tratados con IDPP4 mostraron un mayor cumplimiento terapéutico, mejor control metabólico y menor proporción de hipoglucemias en comparación con los otros grupos comparados. En la bibliografía consultada, los estudios publicados de cumplimiento y/o persistencia con antidiabéticos orales son escasos y difíciles de comparar entre ellos por la diferente metodología empleada en su medición. Estos estudios muestran un cumplimiento entre el $40-80 \%{ }^{(20-21)}$. Hans ${ }^{(22)}$, en un estudio realizado con un elevado número de pacientes ( $N=108.592$; $E E$. UU.) en tratamiento con metformina, glitazonas y SU en monoterapia con un seguimiento de dos años de duración, observó que el cumplimiento variaba entre un $61,3-73,8 \%$. Rathmann ${ }^{(23)}$, en un reciente estudio realizado con una metodología similar a la de este estudio (diseño retrospectivo, dos años de duración), destaca que los pacientes en tratamiento con IDPP4 en comparación con SU, estos presentan una mejor persistencia al tratamiento antidiabético, asociándose con niveles más bajos de hipoglucemias y un menor riesgo (26\%) de presentar un ECV. Resultados que son consistentes con los del presente trabajo. La explicación más plausible podría encontrarse por la presencia de un mejor perfil de tolerancia y seguridad de los IDPP4, en especial por la presencia de menores tasas de hipoglucemias (propiedades farmacocinéticas y farmacodinámicas) ${ }^{(8,11,24-26)}$; aunque se necesitarían más ensayos clínicos para reforzar la consistencia de estos resultados. Parece claro que el papel de los IDPP4 en el arsenal terapéutico de la DM2 está evolucionando rápidamente, aunque faltan datos a largo plazo que evalúen su efecto sobre el control metabólico y del riesgo cardiovascular (22,23,26,27).

Los pacientes del grupo IDPP4 también mostraron un menor coste sanitario, con una reducción del uso de los recursos en atención especializada que ocasionaron menos ingresos hospitalarios. Los limitados estudios existentes muestran que cuanto mayor sea el cumplimiento y el control metabólico de estos pacientes, menor es el riesgo de hospitalización ${ }^{(22,24)}$. A modo de ejemplo, Breitscheidel ${ }^{28)}$, en una revisión efectuada, concluye que la mejora del cumplimiento puede dar lugar a una reducción de los costes sanitarios totales en DM2; en siete estudios, el cumplimiento se asoció inversamente con los costes sanitarios totales, mientras que se destaca un menor coste debido a una menor proporción de días de hospitalización. No obstante, la variabilidad en los estudios revisados fue alta, circunstancia que dificulta la comparación de los resultados. En general, lo hallado en este estudio es consistente con estos estudios (22,24), destacando la asociación de la hipoglucemia con los costes ${ }^{(10,23,29,30)}$.

Los datos hallados en este estudio también muestran que los pacientes en tratamiento con IDPP4 vs SU presentan, a los dos años de evolución, menores tasas de ECV e insuficiencia renal. En este aspecto, diversos autores han demostrado que un buen control metabólico, representado por valores bajos de $\mathrm{HbA} 1 \mathrm{c}$, produce una mejoría significativa en la incidencia y evolución de las complicaciones vasculares, beneficio que persiste durante años, aunque empeore el control metabólico (24). Dada la estrecha relación que existe entre algunas microangiopatías (fundamentalmente la nefropatía) y los $E C V$, es lógico considerar que un buen control metabólico incluiría positivamente en la misma, pero con una intensidad menor que el control de otros factores de riesgo, como la dislipemia y la hipertensión arterial ${ }^{(1,5)}$. 
En la actualidad, los IDPP4 podrían tener un beneficio cardiovascular (pacientes ancianos y/o en insuficiencia renal), pero deberemos esperar los resultados de diversos ensayos clínicos ya en curso ${ }^{(21,23,27)}$. El coste de los IDPP4 es mayor que las SU, pero se compensa con las repercusiones ocasionadas por las complicaciones (hipoglucemias, macrovasculares).

Existe un cierto grado de controversia en la eficacia clínica y el coste-efectividad de estos fármacos en la literatura revisada. En este sentido, Karagiannis ${ }^{(32)}$, en una revisión sistemática efectuada sobre la base de 19 estudios ( $N=7136$, IDPP4 y $N=6745$, otros fármacos hipoglucemiantes), concluyó que los IDPP4 frente a SU/ pioglitazona pueden reducir de forma similar la $\mathrm{HbA} 1 \mathrm{c}$; aunque el estudio manifiesta una cierta incertidumbre en la seguridad a largo plazo, que también deben tenerse en cuenta. Por su parte, el estudio de Klarenbach (33), detalla que para la mayoría de los pacientes con DM2 que no obtienen los objetivos terapéuticos de control con metformina en monoterapia, la inclusión de una SU representa la terapia de segunda línea más rentable.

En este artículo se muestran las limitaciones propias de los estudios retrospectivos, como por ejemplo, el subregistro de la enfermedad o la posible variabilidad de los profesionales y pacientes, al ser un diseño observacional. Cabe destacar que este tipo de diseños no está exento de sesgos (factores no tenidos en cuenta como el nivel económico-social, cultural o de escolarización, las dosis farmacológicas consumidas o la adecuación terapéutica, por citar algunos ejemplos) que se deberían de minimizar. La principal objeción al estudio es su indudable sesgo de selección por parte del médico responsable a la hora de administrar unos u otros fármacos, por lo que la interpretación de los resultados debe tomarse con prudencia. Otra limitación que debe considerarse es el no haber podido conocer los motivos por los cuales se decidió agregar un segundo medicamento a metformina, además, no se obtuvo el tiempo en tratamiento específico de los pacientes con el segundo medicamento (IDPP4 o SU). También se puede considerar como una limitación del estudio, por su diseño, que las complicaciones vasculares se puedan asociar a un mejor control diabético derivado del uso de IDPP4 puesto que pueden existir otros factores no tenidos en cuenta, que lo pueden condicionar. Otra limitación del estudio se refiere a la medida de la hipoglucemia, puesto que solo se identificaron los episodios en los que el paciente requirió asistencia sanitaria y esta quedó documentada, por lo que podría hacer un infradiagnóstico de los casos. Cabe destacar que el mejor diseño para valorar la eficacia del fármaco (adecuado control metabólico: $\mathrm{HbAc1)}$ es un ensayo clínico aleatorio y controlado, donde los pacientes deben ser seleccionados al azar (doble ciego) para evitar los sesgos mencionados con anterioridad. Otra limitación fue que no se pudo medir adherencia en términos adecuados y solo se obtuvo con una "proxy" (cumplimiento terapéutico), por lo que en un diseño experimental (ensayo clínico) se podría también medir la adherencia, puesto que este es un factor que influye en la efectividad de la terapia. Estas dos últimas apreciaciones, deben de interpretarse como las mayores limitaciones del estudio.

Serán necesarias futuras investigaciones para disponer de estudios de coste/efectividad y de demora diagnóstica y de tratamiento, y recabar datos en otras organizaciones sanitarias. En conclusión, a pesar de las limitaciones del estudio, los pacientes en tratamiento con IDPP4 presentaron mayor cumplimiento y control de la diabetes, con menores tasas de hipoglucemias y complicaciones macrovasculares, circunstancia que repercute en unos menores costes sanitarios.

Contribución de los autores: la concepción y el diseño del manuscrito fueron realizados por AS; la recogida de los datos por AS y RN; el análisis estadístico por AS; y la interpretación de los datos, redacción, revisión y aprobación del manuscrito remitido, por todos los autores.

Conflictos de interés: los autores declaran no tener conflictos de interés.

Fuentes de financiamiento: trabajo financiado por Novartis Farmacéutica SA.

\section{REFERENCIAS BIBLIOGRÁFICAS}

1. Inzucchi SE, Bergenstal RM, Buse JB, Diamant $M$, Ferrannini E, Nauck M, et al. Management of hyperglycemia in type 2 diabetes: a patient-centered approach: position statement of the American Diabetes Association (ADA) and the European Association for the
Study of Diabetes (EASD). Diabetes Care. 2012 Jun;35(6):1364-79. doi: $10.2337 / \mathrm{dc} 12-0413$.

2. Weintraub WS, Daniels SR, Burke LE, Franklin BA, Goff DC Jr, Hayman LL, et al. Value of primordial and primary prevention for cardiovascular disease: a policy statement from the American Heart Association. Circulation. 2011 Aug 23;124(8):967-90. doi: 10.1161/ CIR.0b013e3182285a81.

3. Hopper I, Billah B, Skiba M, Krum H. Prevention of diabetes and reduction in major cardiovascular events in studies of subjects with 
prediabetes: meta-analysis of randomised controlled clinical trials. Eur J Cardiovasc Prev Rehabil. 2011 Dec;18(6):813-23. doi: $10.1177 / 1741826711421687$.

4. Gómez Huelgas R, Díez-Espino J, Formiga F, Lafita Tejedor J, Rodríguez Mañas L, González-Sarmiento E, et al. Tratamiento de la diabetes tipo 2 en el paciente anciano. Med Clin (Barc). 2013;140(3):134.e1-134.e12.

5. Menéndez Torre E, Lafita Tejedor J, Artola Menéndez S, Milán NúñezCortés J, Alonso García A, Puig Domingo $\mathrm{M}$, et al. Recommendations for the pharmacologic treatment of hyperglycemia in type 2 diabetes. Consensus document. Nefrologia. 2011;31(1):17-26. doi: 10.3265/ Nefrologia.pre2010.Nov.10715.

6. Nadeau DA. Physiologic and weightfocused treatment strategies for managing type 2 diabetes mellitus: the metformin, glucagon-like peptide-1 receptor agonist, and insulin (MGI) approach. Postgrad Med. 2013 May;125(3):112-26. doi: 10.3810/ pgm.2013.05.2666.

7. George MM, Copeland KC. Current treatment options for type 2 diabetes mellitus in youth: today's realities and lessons from the TODAY study. Curr Diab Rep. 2013 Feb;13(1):72-80. doi: 10.1007/s11892-012-0334-z.

8. Imam K. Management and treatment of diabetes mellitus. Adv Exp Med Biol. 2012;771:356-80.

9. Seaquist ER, Anderson J, Childs B, Cryer P, Dagogo-Jack S, Fish L, et al. Hypoglycemia and diabetes: a report of a workgroup of the American Diabetes Association and the Endocrine Society. J Clin Endocrinol Metab. 2013 May;98(5):1845-59. doi: 10.1210/ jc.2012-4127.

10. Liu SC, Tu YK, Chien MN, Chien KL. Effect of antidiabetic agents added to metformin on glycaemic control, hypoglycaemia and weight change in patients with type 2 diabetes: a network meta-analysis. Diabetes Obes Metab. 2012 Sep;14(9):810-20. doi: 10.1111/j.1463-1326.2012.01606.x.

11. Rungby J. Inhibition of dipeptidyl peptidase 4 by BI-1356, a new drug for the treatment of beta-cell failure in type 2 diabetes. Expert Opin Investig Drugs. 2009 Jun;18(6):835-8. doi: $10.1517 / 13543780902953707$.
12. Tucker DM, Palmer AJ. The costeffectiveness of interventions in diabetes: a review of published economic evaluations in the UK setting, with an eye on the future. Prim Care Diabetes. 2011 Apr;5(1):9-17. doi: 10.1016/j. pcd.2010.10.001.

13. Li R, Zhang P, Barker LE, Chowdhury FM, Zhang X. Cost-effectiveness of interventions to prevent and control diabetes mellitus: a systematic review. Diabetes Care. 2010 Aug;33(8):187294. doi: $10.2337 / \mathrm{dc} 10-0843$.

14. Lamberts H, Wood M, HofmansOkkes ÍM, editores. The Ínternational Classification of Primary Care in the European Community. with a multi-language layer. Oxford: Oxford University Press; 1993.

15. Charlson ME, Pompei P, Ales KL, Mackenzie CR. A new method of classifying prognostic comorbidity in longitudinal studies: development and validation. J Chronic Dis. 1987;40(5):373-83.

16. World Health Organization. The Anatomical Therapeutic Chemical Classification System. Geneva: WHO; 1991.

17. Peterson AM, Nau DP, Cramer JA, Benner J, Gwadry-Shidar F, Nichol M. A checklist por medication compliance and persistence studies using retrospective databases. Value Health. 2007 Jan-Feb;10(1):3-12.

18. Instituto Nacional de Estadística. Encuesta anual de costes laborales del año 2010 [Internet]. Madrid: INE; 2011 [citado en setiembre de 2012]. Disponible en: http://www.ine.es/ prensa/np671.pdf

19. Thompson SG, Barber JA. How should cost data in pragmatic randomised trials be analysed? BMJ. 2000 Apr 29;320(7243):1197-200.

20. Reach G. [Non-adherence to therapy in type 2 diabetes]. Presse Med. 2013 May;42(5):886-92. doi: 10.1016/j. lpm.2013.02.314. [Artículo en Frances].

21. Loke YK, Hinz I, Wang X, Salter C. Systematic review of consistency between adherence to cardiovascular or diabetes medication and health literacy in older adults. Ann Pharmacother. 2012 Jun;46(6):863-72. doi: 10.1345/ aph.1Q718.

22. Hansen RA, Farley JF, Droege M, Maciejewski ML. A retrospective cohort study of economic outcomes and adherence to monotherapy with metformin, pioglitazone, or a sulfonylurea among patients with type 2 diabetes mellitus in the United States from 2003 to 2005. Clin Ther. 2010 Jul;32(7):1308-19. doi: 10.1016/j. clinthera.2010.07.011.

23. Rathmann W, Kostev K, Gruenberger JB, Dworak M, Bader G, Giani G. Treatment persistence, hypoglycaemia and clinical outcomes in type 2 diabetes patients with dipeptidyl peptidase- 4 inhibitors and sulphonylureas: a primary care database analysis. Diabetes Obes Metab. 2013 Jan;15(1):55-61. doi: 10.1111/j.1463-1326.2012.01674.x.

24. Kim HS, Shin JA, Lee SH, Kim ES, Cho $\mathrm{JH}$, Son HY, et al. A comparative study of the effects of a dipeptidyl peptidaseIV inhibitor and sulfonylurea on glucose variability in patients with type 2 diabetes with inadequate glycemic control on metformin. Diabetes Technol Ther. 2013 Oct;15(10):810-6. doi: 10.1089/dia.2013.0038.

25. Erlich DR, Slawson DC, Shaughnessy A. Diabetes update: new drugs to manage type 2 diabetes. FP Essent. 2013 May;408:20-4.

26. Mathieu C, Barnett $\mathrm{AH}$, Brath $\mathrm{H}$, Conget I, de Castro JJ, Göke R, et al. Effectiveness and tolerability of secondline therapy with vildagliptin vs. other oral agents in type 2 diabetes: a real-life worldwide observational study (EDGE). Int J Clin Pract. 2013 Oct;67(10):94756. doi: 10.1111/ijcp.12252.

27. Scheen AJ. DPP-4 inhibitors in the management of type 2 diabetes: a critical review of head-to-head trials. Diabetes Metab. 2012 Apr;38(2):89-101. doi: 10.1016/j.diabet.2011.11.001.

28. Breitscheidel L, Stamenitis S, Dippel FW, Schöffski O. Economic impact of compliance to treatment with antidiabetes medication in type 2 diabetes mellitus: a review paper. J Med Econ. 2010 Mar;13(1):8-15. doi: $10.3111 / 13696990903479199$.

29. Guillausseau PJ. Impact of compliance with oral antihyperglycemic agents on health outcomes in type 2 diabetes mellitus: a focus on frequency of administration. Treat Endocrinol. 2005;4(3):167-75.

30. Wild $\mathrm{H}$. The economic rationale for adherence in the treatment of type 2 diabetes mellitus. Am J Manag Care. 2012;18(3 Suppl):S43-8. 
31. Jermendy G, Wittmann I, Nagy L, Kiss Z, Rokszin G, Abonyi-Tóth Z, et al. Persistence of initial oral antidiabetic treatment in patients with type 2 diabetes mellitus. Med Sci Monit. 2012 Feb;18(2):CR72-77.

32. Karagiannis T, Paschos P, Paletas K, Matthews DR, Tsapas A. Dipeptidyl peptidase- 4 inhibitors for treatment of type 2 diabetes mellitus in the clinical setting: systematic review and metaanalysis. BMJ. 2012 Mar 12;344:e1369. doi: 10.1136/bmj.e1369.

33. Klarenbach S, Cameron C, Singh S, Ur E. Cost-effectiveness of secondline antihyperglycemic therapy in patients with type 2 diabetes mellitus inadequately controlled on metformin.
CMAJ. 2011 Nov 8;183(16):E1213-20. doi: $10.1503 /$ cmaj.110178.

Correspondencia: Antoni Sicras Mainar

Dirección: Dirección de Planificación. C. Gaietà Soler, 6-8 entlo. 08911 Badalona. Barcelona. España

Teléfono: 0034935072684.

Correoelectrónico:asicras@bsa.cat

Visite nuestra página en Facebook, www.facebook.com/rpmesp Infórmese sobre los eventos y los nuevos contenidos de la Revista Peruana de Medicina Experimental y Salud Pública

\section{REVISTA PERUANA DE MEDICINA EXPERIMENTAL Y SALUD PÚBLICA} Difundiendo investigación en salud

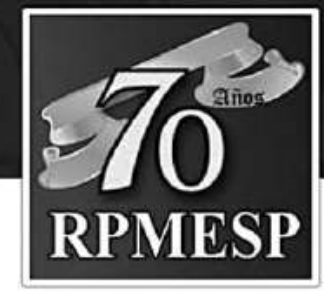
1942 - 2012

Comunidad

La Revista Peruana de Medicina Experimental y Salud Publica es el órgano oficial de difusión cientifica del Instituto Nacional de Salud

\section{Revista Peruana de} Medicina Experimental y Salud Pública (OFICIAL) A 1254 personas les gusta esta página $\cdot 8$ personas están
hablando de esto

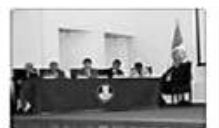

Fotos

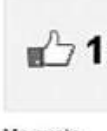

1254

Me gusta

COMENTARIOS DE SIMPOSIO SALUD El simposio de verdar estuvo muy bueno. $M$ Notas 5 